\title{
Fractal dimension analysis with popcorn grains and popped popcorn grains
}

\author{
Eduardo do Carmo*1@, Marcelo Goncalves Hönnicke ${ }^{1 @}$ \\ ${ }^{1}$ Universidade Federal da Integração Latino-Americana, Instituto de Ciências da Vida e da Natureza, \\ Foz do Iguacu, PR, Brasil.
}

Received on March 24, 2021. Revised on June 17, 2021. Accepted on August 10, 2021.

\begin{abstract}
In this manuscript, a very simple experiment was mounted to explore the fractal dimension of a popcorn grain before (popcorn grains) and after (popcorn itself) the popping process.

Keywords: fractal dimension, popcorn.
\end{abstract}

\section{Introduction}

A fractal [1] is a mathematical term that indicates the classes of complex geometric shapes which are not Euclidian, i.e., do not show conventional spatial dimensions ( 1 or 2 or 3 ). The fractal dimension is different from the Euclidean dimension in the way that it indicates, at which level, the object shape is far from the uniformity. Different methodologies have been proposed to introduce the concept of fractal to students such as, two dimensional picture analysis with open source software [2], analysis of structures found in the nature [3], analysis of man-made procedures [4] and analysis of dedicated [7/9] and sample modeling experiments [10]. In this sense, one can imagine that by adapting the simple methodology used to analyze the fractal dimension of a cauliflower [3] and different types of breads [4, 5] as well as the pore space in sponges [6] and even, the fractal dimension of different types of experiments involving crumpled paper balls [7 [9] and aggregates of tennis balls [10, it is also possible to obtain the fractal dimension of the popcorn grains before and after the popping process.

In this work, the fractal analysis was made by determining the fractal dimension of the popcorn grains before and after the popping process. Five different sets of popcorn grains with different quantities were analyzed.

\section{Fractal Dimension Experiment and Analysis}

In order to obtain the fractal dimension before and after the popping process, we decided to pop five different sets of popcorn grains with different grain amounts (ranging

\footnotetext{
${ }^{*}$ Correspondence email address: eduardo.carmo@unila.edu.br
}

from 50 to 800) (Fig. 1, Table 1). The popcorn grains were popped by using an aluminum stovetop popcorn popper. For each different set, the total masses and total volumes of the popcorn grains were determined before $\left(m_{1}-m_{5}\right.$ and $\left.V_{1}-V_{5}\right)$ and after the popping process $\left(m_{1}^{\prime}-m_{5}^{\prime}\right.$ and $\left.V_{1}^{\prime}-V_{5}^{\prime}\right)$ (Table 1). The methodology employed here was similar to the one used to determine the fractal dimension of the pore space in sponges [6]. In that work, the void spaces (pore spaces) were filled with water in order to determine the pores water-mass equivalent. Such values were then matched with different sponge cube edges in order to determine the fractal dimension of the pore space. By analogy, here, the waterfilled pores can be represented by the popcorn grains and the popcorns, while the sponge material filled space can be represented by the void air spaces. Then, the popcorn grains and popcorns masses $\left(m_{1}-m_{5}\right.$ and $\left.m_{1}^{\prime}-m_{5}^{\prime}\right)$ are matched with five different cube edges $\left(a_{1}-a_{5}\right.$ and $\left.a_{1}^{\prime}-a_{5}^{\prime}\right)$ with volumes equivalent to the measured volumes of the five different sets $\left(V_{1}-V_{5}\right.$ and $\left.V_{1}^{\prime}-V_{5}^{\prime}\right)$.

A basic way to characterize a fractal is by the fractal dimension $(D)$, also called the Hausdorff dimension. Mandelbrot 11 suggested that a fractal may be defined as an object which has a Hausdorff dimension different from its topological dimension. For example, the Cantor set [1], has topological dimension of zero and $D \sim 0.63$, i.e., it is set between a dot and a line. For a solid threedimensional body (e.g., the crumpled paper balls [7]), $D$ between 2 and 3, can then represent the "dimensions" of the surfaces of these bodies, which appear rough surfaces between the smooth two dimensional surface and the three dimensional solid. The $D$ of an object can be found by matching its mass $(m)$ with a characteristic length (a), so that:

$$
a \propto m^{\frac{1}{D}}
$$

Since our measurements are based on total quantities of masses and volumes (Table 1), mass-volume equivalent 

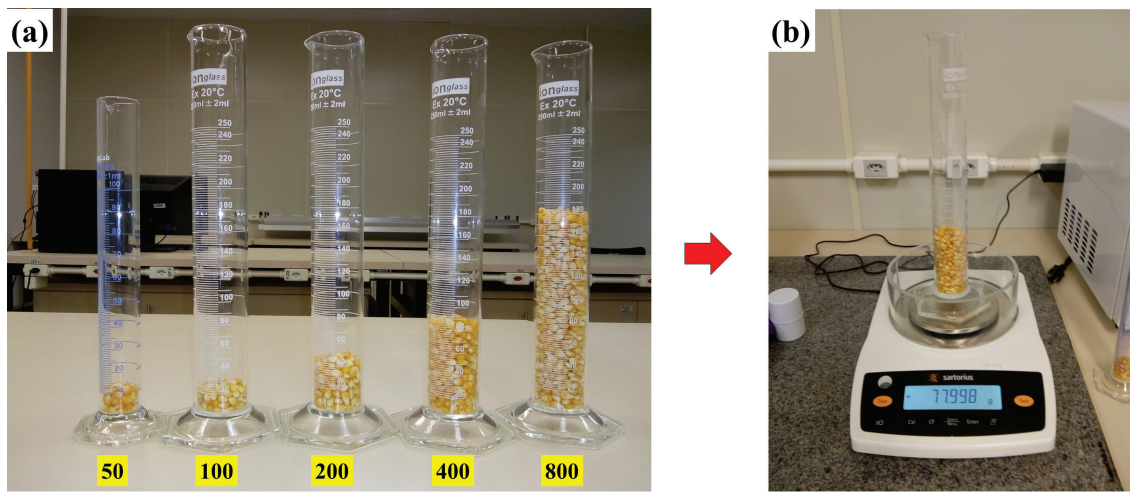

(c)
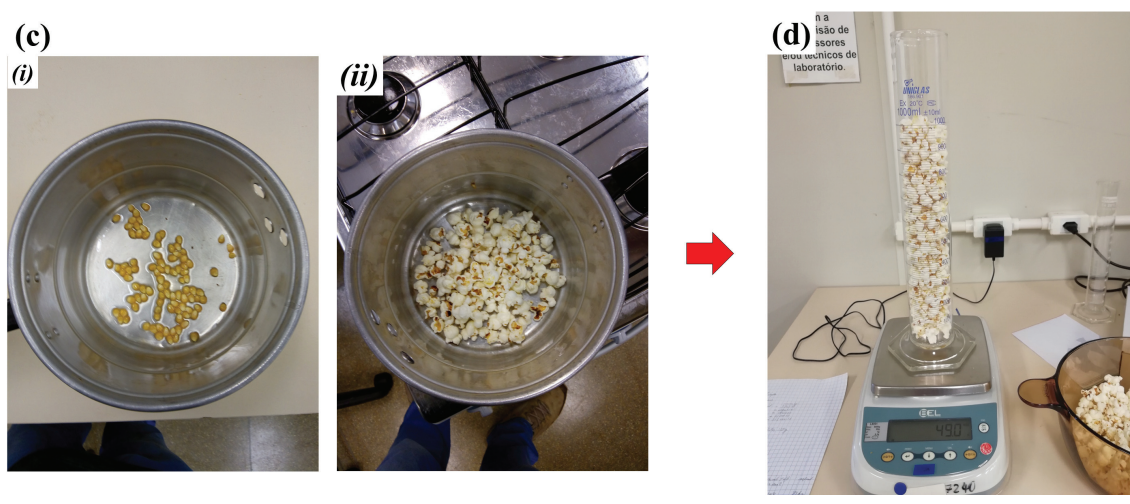

Figure 1: Representation of the procedure employed to determine the initial (before popping) and final (just after popping) physical constants (volume and mass) of the different sets of popcorn grains (Zea Mays L.) with different grain amounts in order to determine the fractal dimension. (a) Initial volume determination of the 5 different sets, used in the experiment, with the popcorn grain amounts ranging from 50 (first set) to 800 (last set). (b) Mass determination procedure (by using a digital scale). As an example, the 4th set with 400 popcorn grains is shown. (c) Popping procedure: (i) Popcorn grains embedded in soybean oil inside a pan previous to the popping process and; (ii) Popped popcorn grains (popcorn itself) inside the pan just after being popped (in specifics, the 2nd set with 100 popcorn grains). (d) Final volume and mass determination of the popcorn (in specifics, part of the 5 th set with 800 popcorn grains).

Table 1: Masses and volumes of the five different experiment sets (with different grains amount) measured to determine the fractal dimension $(D)$ of the popcorn grains (before the popping process) and of the popped popcorn grains (after the popping process popcorn itself). Cube edges for the mass-volume equivalent cubes are also shown. The errors in the masses and in the volumes are given by the measuring instruments (last digit of the digital scale and half of the smallest division of the graduated cylinders, respectively). The errors in the cube edges are given by the propagation of uncertainties of the measured volumes.

\begin{tabular}{|c|c|c|c|c|c|c|}
\hline $\begin{array}{l}\text { Popcorn } \\
\text { grains } \\
\text { amount }\end{array}$ & $\begin{array}{c}\text { Total masses } \\
\left(m_{1}-m_{5}\right) \\
\text { before } \\
\text { popping }(\mathrm{g})\end{array}$ & $\begin{array}{c}\text { Total masses } \\
\left(m_{1}^{\prime}-m_{5}^{\prime}\right) \\
\text { after popping } \\
(\mathrm{g})\end{array}$ & $\begin{array}{l}\text { Total volumes } \\
\left(V_{1}-V_{5}\right) \text { before } \\
\text { popping }\left(\mathrm{cm}^{3}\right)\end{array}$ & $\begin{array}{c}\text { Cube edges } \\
\left(a_{1}-a_{5}\right) \\
\text { before } \\
\text { popping }(\mathrm{cm})\end{array}$ & $\begin{array}{l}\text { Total volumes } \\
\left(V_{1}^{\prime}-V_{5}^{\prime}\right) \text { after } \\
\text { popping }\left(\mathrm{cm}^{3}\right)\end{array}$ & $\begin{array}{l}\text { Cube edges } \\
\left(a_{1}^{\prime}-a_{5}^{\prime}\right) \\
\text { after popping } \\
\quad(\mathrm{cm})\end{array}$ \\
\hline 50 & $9.2 \pm 0.1$ & $10.5 \pm 0.1$ & $11 \pm 2$ & $2.2 \pm 0.1$ & $120 \pm 10$ & $4.93 \pm 0.14$ \\
\hline 100 & $18.2 \pm 0.1$ & $22.1 \pm 0.1$ & $22 \pm 2$ & $2.8 \pm 0.1$ & $320 \pm 20$ & $6.84 \pm 0.14$ \\
\hline 200 & $36.7 \pm 0.1$ & $44.0 \pm 0.1$ & $42 \pm 2$ & $3.5 \pm 0.1$ & $780 \pm 20$ & $9.21 \pm 0.08$ \\
\hline 400 & $71.5 \pm 0.1$ & $85.7 \pm 0.1$ & $88 \pm 2$ & $4.4 \pm 0.0$ & $2320 \pm 60$ & $13.24 \pm 0.08$ \\
\hline 800 & $139.6 \pm 0.1$ & $168.4 \pm 0.1$ & $160 \pm 2$ & $5.43 \pm 0.02$ & $3770 \pm 60$ & $15.56 \pm 0.11$ \\
\hline
\end{tabular}

cubes were defined, for each different set, with the same measured masses and volumes (Fig. 2). Then, for each different set, it was possible to determine different cube edges (characteristic length) (Fig. 2). Therefore, through a log-log plot of the different cube masses $\left(m_{1}-m_{5}\right.$ and $\left.m_{1}^{\prime}-m_{5}^{\prime}\right)$ versus different cube edges $\left(a_{1}-a_{5}\right.$ and $\left.a_{1}^{\prime}-a_{5}^{\prime}\right)$ (Fig. 3), it was possible to determine the fractal dimensions $(D s)$ of the popcorn 
(a)
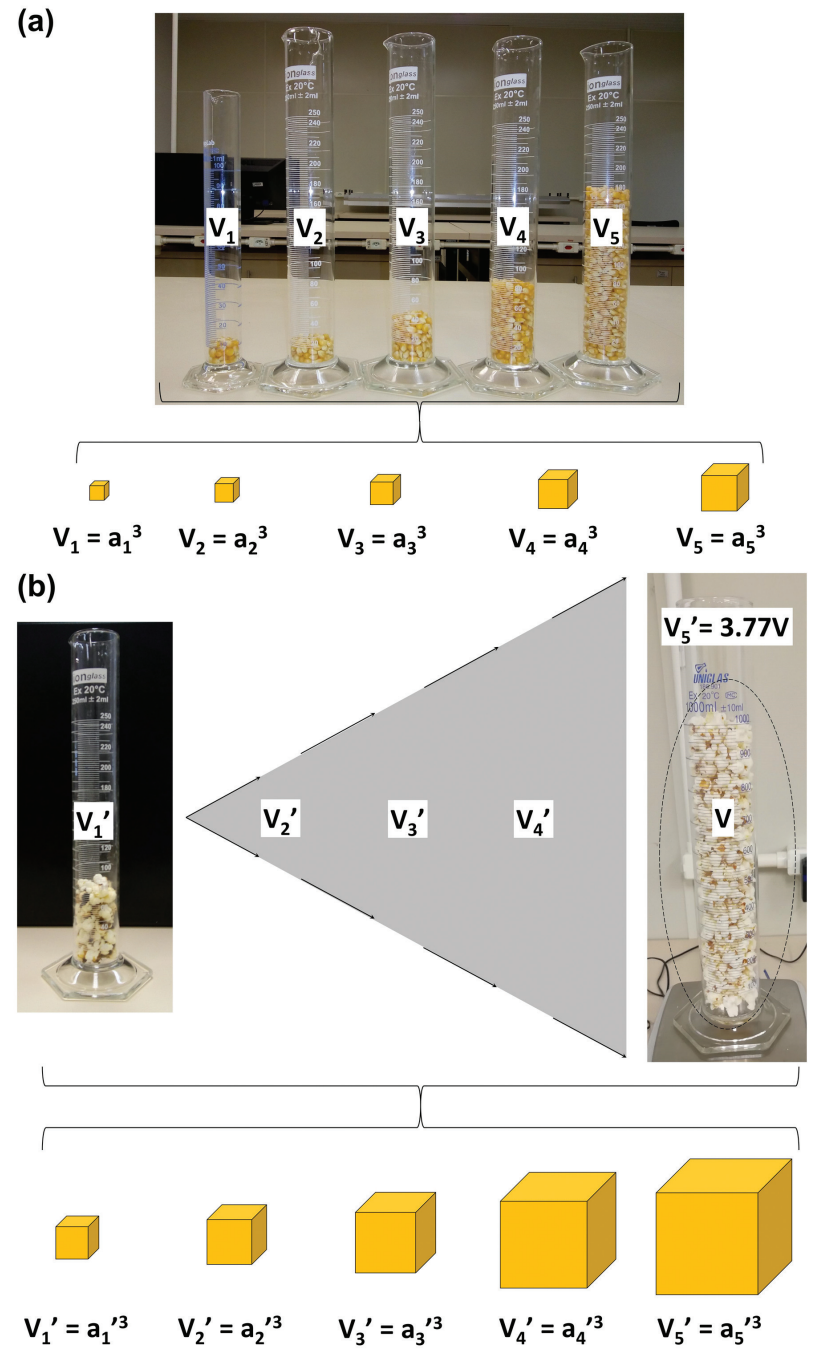

Figure 2: Procedure employed to find the characteristic lengths. The volumes measured, for each different set for the popcorn grains (a) and the popped popcorn grains (popcorn itself) (b), were matched with cubes with the same volumes (mass-volume equivalent cubes): $V_{1}-V_{5}$, for the popcorn grains and $V_{1}^{\prime}-V_{5}^{\prime}$, for the popcorns. Each different cube, corresponding to each different measured volume, has a different characteristic length (cube edges: $a_{1}-a_{5}$ for the popcorn grains and; $a_{1}^{\prime}-a_{5}^{\prime}$ for the popcorns). For the popcorn volumes (b), since we missed pictures, the measured volumes $V_{2}^{\prime}-V_{4}^{\prime}$ on the graduated cylinders are not shown. The volume $V_{5}^{\prime}$ correspond to 3.77 times the volume shown in the picture. All the schematic cubes are shown in relative scale, i.e., for example, $a_{5} \sim 2.5 a_{1}$, $a_{5}^{\prime} \sim 3.16 a_{1}^{\prime}$ and, consequently, $a_{5}^{\prime} \sim 7.07 a_{1}$.

grains as well as of the popcorns. The $D s$ are given by the angular coefficient of the lines those fit the data. The errors were calculated based on the propagation of uncertainties of the measuring instruments (for the masses, the last digit of the digital scale and; for the volumes, half of the smallest division of the different graduated cylinders, respectively) as well as by the linear regression. As previously mentioned, the cube edges were obtained from the mass-volume equivalent cubes (a)

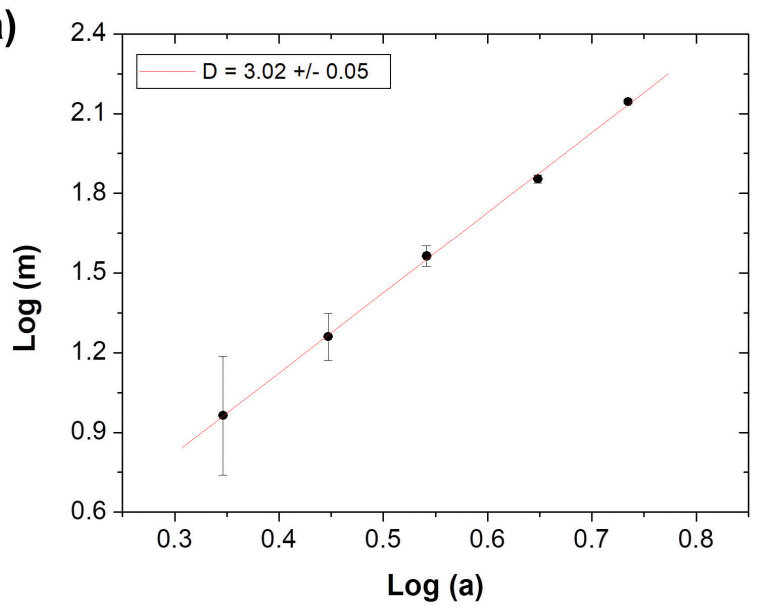

(b)

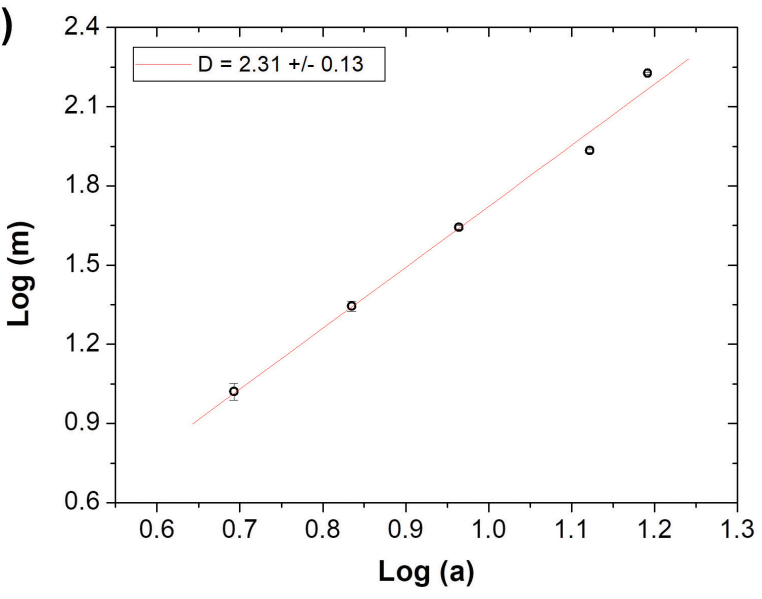

Figure 3: Log-log plot of the different masses $\left(m_{1}-m_{5}\right.$ and $m_{1}^{\prime}-m_{5}^{\prime}$, for the popcorn grains and popcorns, respectively, in grams) versus the different mass-volume equivalent cube edges $\left(a_{1}-a_{5}\right.$ and $a_{1}^{\prime}-a_{5}^{\prime}$, for the popcorn grains and popcorns, respectively, in $\mathrm{cm}$ ) obtained from each different experiment set (five in total, with popcorn grain amounts varying from 50 to 800 - Table 1). (a) Before the popping process (popcorn grains). (b) After the popping process (popped popcorn grains, i.e., popcorn itself). Filled and opened circles are the experimental data, the continuous lines (in red) are the fitted data from which the fractal dimension $(D)$ is extracted. Error bars are also shown. Where they do not appear they are smaller or, at the same size, of the circle representing the experimental data.

so that, their errors were calculated by the propagation of uncertainties of the measured volumes. The fractal dimension of the popcorn grain was found to be $D=$ $3.02 \pm 0.05$, while for the popcorn was found to be $D=2.31 \pm 0.13$. Before popping, the popcorn grain is very regular, which causes an almost perfect fill on the mass-equivalent cube volume. Then, it is expected that the fractal dimension must be very close to the Euclidian dimension. i.e., $D=3$. After the popping process, with the new strange/wrinkled format, the fractal dimension must be different form the topological dimension.

At last, note that, in the fractal dimension analysis, we have not set apart the part of the soybean oil mass which remains attached to the popcorn grain after it 
pops (please, see, for instance in Table 1 that the mass of a kernel is increased after popping). This seems to not affect significantly our $D$ values. We have made the same analysis based on the log-log plot of the popcorn grains/popcorn quantities versus the cube edges. Based on this analysis, the fractal dimensions $D s$ of the popcorn grain as well as the popcorn were found to be $3.07 \pm 0.05$ and $2.32 \pm 0.14$, respectively.

\section{Conclusion}

In this work the fractal analysis of the popcorn grains was investigated by the fractal dimension $(D)$ of popcorn grains and popped popcorn grains (popcorn itself). The $D$ s of the popcorn grain surface as well as of the popcorn surface were found to be $D=3.02 \pm 0.05$ and $D=2.31 \pm 0.14$, respectively. Both results were justified. The popcorn grain is very regular then its fractal dimension must be close to $D=3$ (Euclidian dimension). Otherwise, the popcorn with the wrinkled format should have a fractal dimension different form the topological one. The idea was to show how such a quotidian event, i.e., popping popcorn grains, could be used to illustrate the fractal analysis in a very simple way.

\section{Acknowledgments}

The authors acknowledge Mr. Wagner Ferreira for the help in providing the stove used in this experiment.

\section{References}

[1] B.B. Mandelbrot, The Fractal Geometry of Nature (W.H. Freeman \& Co., New York, 1983).

[2] P.V.S. Souza, R.L. Alves and W.F. Balthazar, The Physics Teacher 57, 467 (2019).

[3] M. Zanoni, The Physics Teacher 40, 18 (2002).

[4] D.H. Esbenshade Jr., The Physics Teacher 29, 236 (1991).

[5] M. Amaku, L.B. Horodynski-Matsushigue and P.R. Pascholati, The Physics Teacher 37, 480 (1999).

[6] L.H.F. Silva and M.T. Yamashita, Eur. J. Physics 30, 135 (2009).

[7] M.A.F. Gomes, Am. J. Physics 55, 649 (1987).

[8] R.H. Ko and C.P. Bean, The Physics Teacher 29, 78 (1991).

[9] F.F. Lima, V.M. Oliveira and M.A.F. Gomes, Am. J. Physics 61, 421 (1993).

[10] J. Sabin, M. Bandín, G. Prieto and F. Sarmiento, Physics Education 44, 499 (2009). 